SEFARAD, vol. 79:2, julio-diciembre 2019, págs. 469-496 ISSN: 0037-0894, https://doi.org/10.3989/sefarad.019-014

VARIA

\title{
Obras filosóficas y de jurisprudencia rabínica de Maimónides: un recorrido por sus ediciones y traducciones*
}

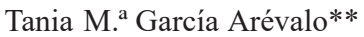

Universidad de Granada

ORCID ID: https://orcid.org/0000-0002-4100-445X

La figura del sabio cordobés Maimónides y la extensa obra que compuso se han convertido en una fuente inagotable de estudio. Sus escritos médicos, aquellos más personales en forma epistolar y su producción filosófica y legal no han cesado de ser analizados desde muy diferentes perspectivas, lo que revierte en la aparición constante nuevos estudios, conclusiones y resultados en torno a ellos. En estas páginas nos centraremos en ofrecer un seguimiento de sus obras filosóficas y de jurisprudencia rabínica a través de las ediciones, traducciones y estudios destacados hasta la fecha con el objetivo de procurar un punto de partida para futuras investigaciones sobre ellas.

Palabras Clave: Moisés ben Maimón; Edad Media; ética; halajá; documentos legales; estudios.

Philosophical and Rabbinical Jurisprudency Works of Maimonides: An OverView through their EDitions and Translations.-The figure of the Cordovan wise Maimonides and the extensive work he composed have become an inexhaustible source of study. His medical writings, those more personal in epistolary form and his philosophical and legal production have not stopped being analyzed from very different perspectives, which reverts in the constant appearance of new studies, conclusions and results around them. In these pages we will focus on offering a follow-up of his philosophical and rabbinic jurisprudence works through the editions, translations and studies highlighted to date in order to provide a starting point for future research on them.

* Estas páginas forman parte de los resultados del Proyecto I+D «Recuperación y estudio del legado lingüístico judeo-árabe de al-Andalus» (FFI2014-51818-P) y del Grupo de Investigación HUM-138 'Hebraístas andaluces'.

**taniagarcia@ugr.es

Copyright: (C) 2019 CSIC. Este es un artículo de acceso abierto distribuido bajo los términos de la licencia de uso y distribución Creative Commons Reconocimiento 4.0 Internacional (CC BY 4.0). 
KeYwords: Moses ben Maimon; Midle Ages; Ethics; Halakhah; Legal documents; Studies.

\section{INTRODUCCIÓN}

Moisés ben Maimón, conocido como Maimónides o por su acrónimo ha-RaMBaM (Córdoba 1138 - Fustat 1204), es una de las personalidades cuyo legado ha traspasado localizaciones geográficas y siglos. Enfrentarse a su producción no es fácil, más aún si tenemos en cuenta todas las facetas en las que Maimónides jugó como intelectual y cuyos resultados se materializaron en numerosas obras. Hablamos de trabajos médicos y farmacológicos ${ }^{1}$; de tratados breves y epístolas ${ }^{2}$, en los que trata de dar respuesta a cuestiones de tipo ético o filosófico y se muestra el

${ }^{1}$ Fred Rosner, Maimonides Medical Writings (Wiesbaden: Kommissionsverlag Franz Steiner, 1984-1989); Gerrit Bos, Maimonides. On the Elucidation of Some Symptoms and the Response to them (Formerly Known as On the Causes of Symptoms) (Leiden-Boston: Brill, 2019); del mismo autor que se ocupa de las ediciones críticas de las traducciones hebreas medievales y Michael R. McVAugh que se ocupa de las latinas, Maimonides. On the Regimen of Health. A New Parallel Arabic-English Translation (Leiden-Boston: Brill, 2019); también de Bos es la edición, traducción y anotación de los aforismos médicos de Maimónides (Maimonides. Medical Aphorisms): tratados 1-5 (Provo: Brigham Young University Press, 2004), tratados 6-9 (Provo: Brigham Young University Press, 2007), tratados 10-15 (Provo: Brigham Young University Press, 2010), tratados 16-21 (Provo: Brigham Young University Press), tratados 22-25 (Provo: Brigham Young University Press, 2015); Maimonides. On Asthma, vol. 1 (Provo: Brigham Young University Press, 2001); con Michael R. McVaugh, On Asthma, vol. 2. Critical editions of Hebrew and Latin translations (Provo: Brigham Young University Press, 2008); con McVaugh, On Hemorrhoids. A new Parallel Arabic-English Edition and Translation (Provo: Brigham Young University Press, 2012); de ambos igualmente, Maimonides. On Poisons and the protection against lethal drugs. A Parallel Arabic-English Edition (Provo: Brigham Young University Press, 2009); Gerrit Bos, (ed.), y Y. Tzvi LangerMANN, (trad.), On rules regarding the practical part of the Medical Art. A Parallel English-Arabic Edition and Translation (Provo: Brigham Young University Press, 2014).

2 Abraham Lichtenberg, (ed.), Collection of the Response of Maimonides and his Letters (Leipzig: HL Shnoys, 1859, reimpr. Farnborough: Gregg, 1969 [en hebreo]); Yosef QAFIH, Maimonides. Letters (Jerusalem: Mossad Harav Kook, 1987 [en hebreo]). Para un estudio de las ediciones y traducciones de las cartas de Maimónides, véase Tania M. ${ }^{a}$ García ArÉvalo, «Cartas de Maimónides: guía de ediciones, traducciones y estudios», Miscelánea de Estudios Árabes y Hebraicos, Sección Hebreo 58 (2009) págs. 87-111. 
tono más distendido y humano de Maimónides, y a aquellos de corte filosófico y de jurisprudencia rabínica que aquí nos ocupan. Si acotamos el análisis al ámbito de estos últimos, no todas las obras del cordobés han gozado de la misma recepción ni, por tanto, de la misma popularidad, siendo testigo de ello su número de ediciones y traducciones. Esto supone que, a la hora de elaborar un estudio bibliográfico, podamos intuir ciertos vacíos de tipo material que responden a que las grandes obras han acaparado el protagonismo frente a otras de tipo menor. No es de extrañar, por tanto, que aquellas de mayor impacto sean con las que Maimónides alcanzó una fama, influencia y respeto más allá de su muerte: el Dalālat al-Hāirīn o Moré Nebujim o 'Guía de Perplejos' y el Mišné Torá o 'Segunda ley', dos corpus que incluso hoy día siguen generando una cantidad incesante de investigaciones y cuyo catálogo de ediciones y traducciones es amplísimo.

En cuanto al primer corpus, la Guía de Perplejos, junto con la importancia de su temática hemos de hacer alusión también a su trabajo de traducción de mano de Šemuel ibn Tibbón en vida de Maimónides. En ella, Maimónides utiliza fuentes muy variadas, desde la filosofía aristotélica a la ciencia de la época, el pensamiento árabe y literatura rabínica, lo que dará como fruto una obra de gran profundidad. El trabajo no será fácil e incluso tendrá que oponerse a los ataques de los cabalistas y tradicionalistas hebreos, dando pie a una enorme polémica que continuará hasta mucho después de su muerte ${ }^{3}$. Sin embargo, la labor de Ibn Tibbón es destacable, no solo para entender su trascendencia sino también los problemas con los que hubo de enfrentarse el traductor.

Sobre el Mišné Torá, es un vasto comentario a la ley judía dividido en catorce libros, cada uno de ellos conformado, a su vez, por tratados, capítulos y leyes para hacer comprensibles las prescripciones contenidas en el Talmud. No obstante, la concepción de facilitar el entendimiento de ciertas cuestiones contenidas dentro del ámbito de la religión, la de justificar sus propias argumentaciones de manera unilateral o ideas como la de la resurrección no tardaron en encontrar oposición en otros círculos que lo sentían como una amenaza que pudiera ocasionar que se dejara

3 Olga GonzÁLEz GonzÁLEZ, «Maimónides y la epístola a Ibn Tibbon. Consideraciones traductológicas», en $V$ Encuentros Complutenses en torno a la traducción, ed. Rafael Martín Gaitero (Madrid: Editorial Complutense, 1995) págs. 327-331: 328. 
a un lado el estudio del Talmud mismo, de hecho, el Mišné Torá se convirtió en uno de los códigos legales fundamentales. Así surge la que se ha denominado como la 'controversia maimonideana' o 'anti-maimonideana' que comienza en vida de Maimónides y que continúa vigente muchos siglos después ${ }^{4}$.

La cuestión de la lengua es relevante pues el Mišné Torá fue el único trabajo escrito originalmente en hebreo ${ }^{5}$ y Maimónides mismo se lamentó de no haber preparado versiones hebreas de los demás. Es más, en una carta que envía a uno de sus discípulos, Yosef ibn Gabir ${ }^{6}$, en 1191 en la que le solicitaba que tradujera este corpus al árabe, responde que hubiera perdido su carácter específico y que sí le hubiera gustado traducir todas sus obras al hebreo, incluso, cuando los sabios de Lunel en otra carta le instan a hacer lo mismo con la Guía de Perplejos, él responde que ojalá fuera lo suficientemente joven para poder llevarlo a cabo $^{7}$. De esta carta hay que hacer una especificación ya que Maimónides envía por separado dos misivas, una a Rabí Yonatán ha-Cohen en 1199, jefe de la comunidad de Lunel, y otra general a los sabios de esta ciudad un año más tarde, en 1200. En ambas anuncia que la tercera parte

${ }^{4}$ Isadore Twersky, «Beginnings of Mishneh Torah Criticism», en Biblical and Other Studies, ed. Alexander Altmann (Cambridge: Harvard University Press, 1967) págs. 95-118; Daniel Jeremy SiLver, Maimonidean Criticism and the Maimonidean controversy 1180-1240 (Leiden-Boston: Brill 1965); James Arthur Diamond, Maimonides and the Shaping of the Jewish Canon (Cambridge: Cambridge University Press, 2014); Haim Hillel Ben-Sasson, Raphael Jospe y Dov Schwartz, «Maimonidean Controversy», en Encyclopedia Judaica, eds. Michael Berendaum y Fred Skolnik, vol. 13 (2. ${ }^{a}$ ed., Leiden-Boston: Brill, 2010) págs. 371-381; Norman RotH, «Las reacciones rabínicas ante Maimónides», en Maimónides y su época, coords. Carlos DEL Valle Rodríguez et al. (Madrid: Sociedad Estatal de Conmemoraciones Culturales S. A., 2007) págs. 69-86.

5 Sobre el hebreo de Maimónides, véase Haseeb SHehadeH, «The Arabic Component in Maimonides' Hebrew», en Verbus et Calamus: Semitic and Related Studies in Honour of the Sixtieth Birthday of Professor Tapani Harviainen, eds. Hanuu JuU-Sola et al. (Helsinki: The Finnish Oriental Society, 2004) págs. 323-340.

${ }^{6}$ Véase el texto original en Abraham Lichtenberg, Collection of the Response, pág. 15; Leon D. Stitskin, (ed.), Letters of Maimonides (New York: Yeshiva University Press, 1977) págs. 86-94.

7 Louis Isaac Rabinowitz, «Maimonides, Moses», en Encyclopedia Judaica, eds. Michael Berenbaum y Fred SkolniK, vol. 13 (2nd Edition: Leiden-Boston: Brill, 2010) págs. 381-385: 385 . 
de la Guía estará por llegar. En la segunda, además, alude a que cualquier consulta pueden hacerla a Šemuel ibn Tibbón debido al mal estado de salud que entonces ya acusaba ${ }^{8}$.

El propósito de estas páginas será el de ofrecer un recorrido por aquellas obras de carácter filosófico y jurídico de Maimónides, entre las que destacan el Moré Nebujim y el Mišné Torá, acompañadas de las ediciones y traducciones que se han llevado a cabo en torno a ellas, desde la etapa medieval hasta la modernidad. Para una mayor claridad, los trabajos aparecen según su nombre original, ordenados por su año de composición y junto con una breve descripción de estos.

Apunto, por otra parte, que este artículo seguiría la estela de estudios bibliográficos como los de Jacob Dienstag, que ya lo hiciera con las cartas y epístolas de Maimónides entre 1961 y 1989 o David Lachterman en 1990 y Batya Ben-Shammai en 1991 de modo general ${ }^{9}$.

\section{Obras FILOSÓFICAS Y DE JURISPRUDENCIA: EDICIONES Y TRADUCCIONES}

\subsection{El Maqāla fì-șinā'at al-mantiq (1158)}

La primera obra filosófica de Maimónides parece ser un tratado de lógica, Maqāla fí-ṣinā'at al-mantiq (Tratado del Arte de la Lógica) en su versión árabe y Milot ha-higayón (Vocabulario de lógica) en la hebrea, redactado en su juventud. Y digo parece porque, aunque la mayor

${ }^{8}$ García Arévalo, Cartas de Maimónides, págs. 98-99. Véanse las ediciones de Alfred Freimann, Responsa (Jerusalem: Mekize Nirdamim, 1934) pág. 58; Y. GoldMANN, (ed.), Iguerot ve-Šeelot ve-Tešubot (Varsovia: Goldmann, 1877 [en hebreo]) pág. 305; Solomon Aaron Wertheimer, «Iguéret Tešubat ha-Rambam le-Rabenu Yonatán mi-Lunel», Séfer Guinzé Yerušaláyim (Jerusalem 1867) págs. 35a-37a. Sobre la carta a ibn Gabir, puede verse en la edición de LichtenBerg, Collection of the response, pág. 88 y en la de B. Lewin-Epstein, (ed.), Iguerot ve-Tešubot ha-Rambam (Jerusalem: Lewin-Epstein Bros., 1953) pág. 89, la general a los sabios de Lunel.

9 David Lachterman, «Maimonidean Studies 1950-1986: A Bibliography», Maimonidean Studies 1 (1990) págs. 197-216; Batya Ben-ShammaI, «Twenty-Five years of Research on Maimonides, Bibliography 1965-1990» [en hebreo], Maimonidean Studies 2 (1991) págs. 17-42. 
parte del mundo académico lo haya aceptado así, algunos pusieron en duda esta atribución. Este es el caso de Herbert A. Davidson.

Davidson argumenta que no hay motivos para sostener que esta obra procediera del cordobés más allá de la aparición de su nombre, algo que no es justificación suficiente para atribuírsela. Efectivamente, explica que el original árabe se encuentra en dos formas: el texto completo en caracteres hebreos y, por otra parte, un poco más de la mitad en judeoárabe. Solo un único manuscrito del judeo-árabe contiene el comienzo del tratado y en el encabezado se nombra a Maimónides como el autor. La primera traducción al hebreo fue hecha alrededor de cincuenta años después de la muerte de Maimónides por Mošé ibn Tibbón y esta es la que se encuentra en casi todos los manuscritos y ediciones impresas. En la mayor parte de los manuscritos de los Tibbón, a Maimónides se le nombra autor, ya sea como parte del título situándolo en el encabezamiento o en las palabras de apertura en la primera frase. Hay otros, sin embargo, que lo sitúan en el colofón. Aun así existen al menos dos manuscritos que no muestran el nombre de Maimónides al principio, un gran número tampoco lo tiene en el colofón y los hay que no responden ni a un modo ni al otro. Como Davidson concluye, tenemos al menos dos manuscritos de la traducción de los Ibn Tibbón que no atribuyen directamente este tratado a Maimónides. Es más, uno de los manuscritos árabes identifica al autor como Yacqub Abu Ishaq ibn Yusuf que puede ser un sinónimo del verdadero autor ${ }^{10}$.

Este tratado es una obra puramente filosófica en la que no hay ninguna traza teológica o de contenido específicamente judío ya que no cita el texto bíblico ni el Talmud y tampoco da ninguna indicación en torno a su identidad religiosa ${ }^{11}$. Esto ya lo sostiene también Davidson pues no

${ }^{10}$ Herbert A. Davidson, Moses Maimonides: The Man and his Works (Oxford: Oxford University Press, 2005) págs. 313-322 donde discute extensamente la atribución de este tratado a Maimónides. De la opinión contraria a Davidson es Joel L. KraEmer quien la expone en «Is there a Text in this Class», Aleph: Historial Studies in Science and Judaism 8 (2008) págs. 268-272. Parte de esta polémica se encuentra recogida en Rafael Dascalu, A Philosopher of Scripture: The Exegesis and Thought of Tanhum haYerushalmi (Leiden-Boston: Brill, 2019) págs. 188-190.

11 Joel L. Kraemer, Maimonides: The Life and World of One of the Civilizations' Greatest Minds (New York: Doubleday, 2008) pág. 70. 
encuentra en él ninguna caracterización judía ${ }^{12}$. Sin embargo, una de las cuestiones más relevantes que expone es la concepción de ciencia política que Maimónides (adoptando el consenso general en cuanto a su atribución) divide en cuatro partes: gobierno propio del individuo; gobierno de la casa; gobierno de la ciudad y gobierno de la nación o naciones ${ }^{13}$. Su nombre, como señala Fernando Díaz Esteban, procede de la aparición al final de cada capítulo de todos los términos técnicos que lo componen ${ }^{14}$.

En los manuscritos de las copias de la versión hebrea, el Milot, la inclusión de esta terminología no es solo una lista de palabras sino que se añade a estas una explicación desde la base de la gramática (capítulos 1-6), silogismos (7 y 8), materias y sus formas y naturaleza (9-12), gramática de nuevo (13), facultades (14) y las artes o ciencias (15) ${ }^{15}$.

Como apuntamos anteriormente, se sostiene que Maimónides compuso este tratado en su juventud, muy probablemente cuando aun se encontraba en tierras de al-Andalus alrededor de 1158. No tardó en aparecer la primera traducción al hebreo por Mošé ibn Tibbón en 1254 que, posteriormente, sirvió de base a la traducción latina de Sebastian Münster ${ }^{16} \mathrm{y}$ que ha sido la que en un mayor número de ocasiones se ha

12 Davidson, Moses Maimonides: The Man and his Works, pág. 314.

${ }^{13}$ Howard Kreisel, Maimonides' Political Thought: Studies in Ethics, Law and the Human Ideal (Albany: Suny Press, 1999) pág. 3.

${ }^{14}$ Fernando Díaz Esteban, «Religión y razón en Maimónides», Revista Española de Filosofia Medieval 7 (2000) págs. 31-43: 32.

15 Michelle M. Hamilton, Beyond Faith: Belief, Morality and Memory in a Fifteenth Century Judeo-Iberian Manuscript (Leiden-Boston: Brill, 2014) págs. 118-120. Unos de los últimos estudios de este tratado en relación con Alfonso de la Torre es el que ofrece Rafael HerRera GuillÉn, «Verdad y mentira en sentido judío ("El Tratado del arte de la lógica" de Maimónides en la "Visión Deleytable")», Anales del seminario de historia de la filosofía 35:3 (2018) págs. 715-727, así como el de Michelle M. HAMILTON, «Para construir la verdad: La lógica como nexo entre la tradición judeo-árabe y la "Visión Deleytable"», Anales del seminario de historia de la filosofía 35:3 (2018) págs. 617-629; Sarah Stroumsa, «On Maimonides and on Logic», Aleph: Historial Studies in Science and Judaism 14 (2014) págs. 259-263.

${ }^{16}$ Logica Sapientis Rabbi Simeonis, per Sebastianum Munsterum Latine iuxta Hebraismum uersa, quae Hebraeorum commentaria legere uolentibus, nontam utilis est quam necesaria (Basileæ: apud Io. Frob., 1527; reimpr. Venecia: Bragadini, 1550, Cremona 1566). 
editado, traducido y estudiado ${ }^{17}$. Otra edición con texto introductorio en latín es la de Samson ha-Kaliri, publicado en Fráncfort en $1761^{18}$ cuya microficha se encuentra en la British Library de Londres y que, al parecer, conserva notas manuscritas de Moses Mendelssohn.

Ya en los siglos XIV y XVI encontramos las traducciones hebreas de Yosef ben Yošúa Vives de Lorca y Ahitub ben Isaac de Palermo ${ }^{19}$, siendo la última la que un mayor interés despertó tras la de ibn Tibbón. Tanto la traducción de Vives como la de Ahitub sirvieron a Israel Efros en 1938 para proponer una edición del texto basada en el manuscrito judeoárabe del tratado con once copias de la traducción hebrea de ibn Tibbón más una de Ahitub y otra de Vives ${ }^{20}$. Coetánea a esta es la edición francesa de Moshe Ventura (1935) ${ }^{21}$ y la de Roland Houde 22 (1958) que recoge parte de la traducción inglesa de Efros. En 1959-1960, Mubahat Türker publica el texto completo en caracteres árabes basándose en dos

17 Véase David H. Baneth, (ed.), Maimonides, Introduction to Logic, in the Hebrew version of Moses ibn Tibbon, edited on the basis of early printed editions with textual and explanatory notes by Leon Rотн collated with the manuscript fragments by the Arabic original (Jerusalem: Society of the Hebrew University Press, 1935; reimpr. Jerusalem: Magnes Press, 1987); Isaac Satanov y David Slucki, (ed.), Explanation of Logic, texto hebreo traducido por M. ibn Tibbón con comentarios de Mordekay Comtino y Moisés Mendelson (Varsovia: D. Slutzki, 1865; reimpr. Berlin 1928).

${ }_{18}$ Logica R. Mosis Maimonidis, cum explicatione R. Samson Kaliri que Censura Amplissimae (Frankurt: Facultatis Philosophiae Academiae Francofurtanae).

19 Uno de los ejemplares de esta traducción puede encontrarse dentro de una colección de varios trabajos dentro de los folios 3a-19. ${ }^{\mathrm{a}}$ en el manuscrito Heb. $8 .^{\circ} 3308$ de la National Library que puede verse en http://web.nli.org.il/sites/NLI/English/digitallibrary/ moreshet_bareshet/rambam/maimonides_writings/Pages/milot_hahigayon.aspx (acceso el 25/02/2019). Véase la siguiente traducción de la obra de Ahitub por Moritz CHAMIZER, «R. Achitub aus Palermo Hebäische Übersetzung der Logica Maimunis», Judaica, Festschrift zu Hermann cohens siebzigstem Geburstage (Berlin: Bruno Cassirer, 1912) págs. 423-456.

${ }^{20}$ Israel Efros, (ed.), «Mosheh ben Maimon. Maimonides' Treatise on Logic. The Original Arabic and the Three Hebrew Translations», en Proceedings of the American Academy for Jewish Research 8 (1937-1938) págs. 1-136, y 34 (1966) págs. 6-34.

${ }^{21}$ Moshe Ventura, Terminologie logique. Édition critique du texte hébreu, arabe, grec, latin, allemande, anglais et français. Edition de l'original arabes des chapitres existants (Paris: Librairie Lipschutz, 1935).

22 Roland Houde, Readings in Logic (Dubuque: William C. Brown, 1958). 
manuscritos de Turquía con una introducción en turco y francés y una traducción también al turco ${ }^{23}$.

Otras traducciones que debemos señalar son la de la de Moses Mendelssohn al alemán en 182824; la de Isaac Levi al italiano a finales del siglo XIX ${ }^{25}$; en Rémi Brague al francés (1996) ${ }^{26}$, seguida de la de edición de Yosef Qafih junto con una traducción al hebreo moderno en $1997^{27}$.

\subsection{El Kitāb al-sirāj (1168)}

Escrito originalmente en judeo-árabe y conocido como Séfer haMa'or en hebreo o Luminar y compuesto alrededor de 1168, el Kitāb al-sirāj se presenta como un comentario a la Mišná con el propósito de explicar sus preceptos y desarrollar sus ramificaciones en la tradición rabínica posterior. Parece que esta obra juega un papel menor en la crítica maimonideana dentro del mundo europeo ya que este protagonismo se le atribuye al Mišné Torá ${ }^{28}$.

Sobre sus versiones hebreas, Šemuel ibn Tibbón tradujo el comentario al tratado Abot $^{29}$ seguido de al-Harizi (1194) que se dedicó a la introducción y a los cinco primeros capítulos de Zeraim. La traducción completa fue obra de diversos autores a lo largo de varias generaciones:

${ }^{23}$ Mubahat TÜRKer, (ed.), «Maqalah fi sina'at al-mantiq», Ankara Universitesi Dil ve TarihCografaya Fakültesi Dergisi 18 (1960) págs. 40-64 y anteriormente en la Review of the Institute of Islamic Studies 3:1-2 (1959-1960) págs. 49-110.

24 Moses Mendelssonn, Logikalische terminologie des Rabbi Moses Maimonides, von Mendelssohn commentirt, uebersetzt und mit einem Wortregister versehen von Salomon Heidelberg (Breslau: Gedruckt bei L. Sulzbach und Sohn, 1828).

25 Isaac Levi, Compendio di Logica (Mantova: Tip. G. Mondovi, 1898).

26 Remi Brague, Traité de logique (Paris: Desclée de Brouwer, 1996).

27 Yosef QAFIH, Be'ur meléjet ha-higayón (Qiryat Ono: Makhon Mishnat ha-Rambam, 1997).

28 SiLver, Maimonidean Criticism and the Maimonidean Controversy, pág. 30.

29 El comentario a Abot se conoce como Šemona Peraquim, Ocho capitulos o Ética de Maimónides. Sobre él puede verse la edición y traducción de Joseph I. GorfinKEL, The Eight Chapters of Maimonides (New York: AMS Press, 1966); en español en Carlos Del VAlle, Maimónides. Ética (Los ocho capitulos) (Córdoba: Aben Ezra Ediciones, 2004). 
Yosef ben Isaac ben Alfawal tradujo Mo'ed ${ }^{30}$; Jacob ibn Abbas se encargó de la traducción de Našim, con la colaboración de Hayim ben Salomon, el médico de Ibn Baka; Salomon ben Yosef ben Jacob, de Zaragoza, tradujo el orden Neziquim, exceptuando el tratado de Abot, previamente traducido por ibn Tibbón. Parte de Neziquim fue también editado y traducido por I. Shailat en 1992, quien se ocupó de la misma tarea con Abot dos años más tarde ${ }^{31}$. La más reciente es la de Michael D. Schwarz (2011) ${ }^{32}$. Nataniel ha-Rofé ben Yose ben Almoní tradujo Qedušim y Tohorot $^{33}$.

Debido a que la obra consta de varios capítulos, muchas de las ediciones que encontramos son también de partes aisladas de tratados que ya en sí mismos poseen entidad propia, sin embargo, apuntamos las generales de Toledano (1950), Sasson y Edelmann (1956). La de Y. Kafih -escrito Qafih o Kafah en algunas publicaciones- (1976) es la más relevante de todas ya que se basa en un autógrafo de Maimónides procedente de la biblioteca de Copenhage pese a que no incluya la totalidad de los sedarim ${ }^{34}$.

Sobre la introducción encontramos la edición de Mordecai Rabinowitz en 1961 que también se ocupó del tratado Abot $^{35}$. Sobre el tratado

\footnotetext{
30 Jonas Simon, Maimonides, M., Alfawal. Perúš ha-Mišnayot le-ha-Rambam (Berlin: Itskovski, 1902).

31 Isaac ShaILAT, The Introductions of Maimonides in the 'Commentary on the Mishnah' (Jerusalem: Ma'aliyot, 1992) [en hebreo], y Introduction to Avot (Jerusalem: Ma'aliyot, 1994) [en hebreo].

32 Michael D. Schwarz, The Eight Chapters: The Introduction to Maimonides' Commentary on Tractate Avot (Jerusalem: Ben Zvi, 2011).

33 Moritz Steinschneider, Die Hebraischen Uebersetzungen des Mittel Alters (Berlin: Kommissionsverlag des Bibliographischen bureaus, 1893; reimpr. Graz: Akademische Verlangsanstalt, 1956) pág. 923; Silver, Maimonidean criticism, pág. 30 nota 2.

34 Yosef QAFIH, (ed.), Commentary on the Mishnah (Jerusalem: Mossad Harav Kook, 1976. 3 vols.) [en hebreo]; Solomon Sassoon y Rafael Edelmann, (ed.), Maimonides commentarius in Mishnam e cocibus Hunt 117 et Pococke 295 in bibliotheca Bodleiana oxonensi servatis et 72-73 bibliothecae sassooniensis Letchwoth (Copenhaguen: Ejnar Munksgaard, 1956. 3 vols.); Baruk Toledano, (ed.), «Perúš ha-Mišnayot le-ha-Rambam 'al-Maséjet Yomá bi-meqoró be- 'arabit im targum 'ibrit», Sinai 27 (1950) págs. 169-196 (texto árabe y versión hebrea).

35 Mordecai D. Rabinowitz, (ed.), Mošé ben Maimón. Commentary on the Mishnah. Introduction (Jerusalem: Mossad Harav Kook, 1961) [en hebreo], y Mošé ben Maimón. Commentary on Avot (Jerusalem: Mossad Harav Kook, 1961) [en hebreo].
} 
Šabat, puede verse la de Simon Hopkins basada en fragmentos de la Guenizá de El Cairo con el original árabe y la traducción hebrea ${ }^{36}$. De Meguilat, con el texto árabe y la versión hebrea dependiente de la traducción de Alfawal se ocupó, en 1901, Behrens ${ }^{37}$. De Sanhedrín es posible observar las ediciones de Weisz (1893), Bleichrode (1904) y Gottlieb (1906) ${ }^{38}$. De Guitín se ocupó Goldberg (1902) ${ }^{39}$ y, de Quidušim, Nurock $^{40}$ en ese mismo año. De Baba Batra, tenemos las ediciones de Sänger (capítulos uno al cuatro) que la acompañó de una traducción al árabe, hebreo y alemán en $1912^{41}$; del resto de capítulos (quinto al décimo), se encargó Inmanuel Lewy en 1907 e igualmente acompañó a su edición con una traducción al alemán ${ }^{42}$. La edición de Tohorot la llevó a cabo por primera vez J. Derenbourg a finales del siglo XIX junto con

36 Simon Hopkins, (ed.), Maimonides' Commentary on Tractate Shabbat. The Draft Commentary according to Autograph Fragments from the Cairo Geniza. Arabic Original and Hebrew Translation (Jerusalem: Ben Zvi, 2002).

37 Siegfried Behrens, (ed.), Mose ben Maimuni's Mischnah-Commentar zum Tractat Megillat nebst der hebraeischen Uebersetzung des Josef Ibn al-Fawwal. Kritische Edition mit Anmerkungen (Frankfurt am Main: Kauffmann, 1901) [texto árabe y versión hebrea].

38 Moritz WeIsz, (ed.), Maimonides' Commentar zum Tractat Sanhedrin (Cap. I-III). Zum ersten Male in arabischen Urtext herausgegeben, mit verbesserter hebraeischer Uebersetzung und erläuternden Anmerkungen versehen (Berlin: Itskovski, 1893) [texto árabe y versión hebrea]; Isadore Bleichrode, (ed. y trad.), Maimonides' Commentar zum Tractat Sanhedrin (Cap.IV-V). Arabischer Urtext mit verbesserter hebraeischer Uebersetzung, deutscher Uebersetzung and Anmerkungen (Berlin: Bleichrode, 1904) [texto árabe y versión hebrea y alemana]; Manuel GotTlieb, (ed.), Mose ben Maimun's Commentar zur Mischnah Tractat Sanhedrin. In neuer hebraeischer Uebersetzung aus dem arabischen Urtext mit pruefenden und erläuternden Anmerkungen (Hannover: Berliner, 1906) [traducción hebrea].

39 Hirsch Goldberg, (trad.), Maimonides' Commentar zum Tractat Gittin (Berlin: Itskovski, 1902) [árabe y hebreo].

40 Aron Bar Nurock, (ed.), Maimonides' Commentar zum Tractat Kiddushin (Berlin: M. Poppelauer, 1902) [edición del texto árabe con traducción hebrea].

41 Jacob SÄNger, (ed.), Moses ben Maimun's Mishnah-Commentar zum Tractat Baba Batra (Cap. I-IV) (Berlin: M. Poppelauer, 1912) [edición del texto y traducción al árabe, hebreo y alemán].

42 Inmanuel Lewy, (ed.), Moses ben Maimun's Mishnah-Commentar zum Tractat Baba Batra (Cap. V-X) (Berlin: M. Poppelauer, 1907) [árabe y alemán]. 
una traducción hebrea ${ }^{43}$ y S. N. Skaist lo hizo con Baba Mesía en una edición crítica de $1949{ }^{44}$.

En cuanto a las traducciones, por orden de aparición, al latín encontramos una en seis volúmenes que fue publicada entre 1699 y $1703^{45}$. De Makot tenemos la traducción hebrea de Barth (1879 y 1880) publicada en Berlín. De Pe'a la de D. Herzog (1894) basada en la traducción de al-Harizi ${ }^{46}$, la de Jalá de S. Bamberger (1895) ${ }^{47}$, de Edujot de M. Bermann (1897) ${ }^{48}$, Bamberger (1891) de Kilahim ${ }^{49}$, de Midot por N. Almoli y J. Fromer (1898) ${ }^{50}$, Ketubot por Leopold Nebenzalh (1905) de

43 Joseph Derenbourg, Commentaire de Mä̈monide sur la Mischnah Seder Tohorot (Berlin: Itvskovski, 1887-1891). Publicado por primera vez en árabe y acompañado de una traducción hebrea.

44 Solomon N. Skaist, (ed.), A critical edition of Maimonides' Commentary to the Mishna, Kitab al-siraj, Tractate 'Baba Metzia' (New York: Yeshiva University, 1949) [texto árabe y traducción hebrea].

45 Guilielmus SuREnHusius, Mischna sive totius Hebraeorum juris rituum, systema cum clarissimorum Rabbinorum Maimonidis et Barteenorae commentariis integris. Quibus accededunt variorum auctorum notae ac versions in eos quos ediderunt codices, latinitate donavit ac ntas illustrativi (Amsterdam: Gerardus \& Jacobus Borstius, 16991703. 6 vols.).

46 David Herzog, Maimonides, Moses, 1135-1204. Commentar zum Tractat Peah, zum ersten Male im arabischen Urtext hrsg. (Berlin: S. Calvary, 1894). Su reseña en The Jewish Quarterly Review por Adolf Neubauer llegó un año más tarde y es sumamente interesante: Adolf NeUBAUER, «Reviewed Work: Arabic Commentary on the Mishnah by Moses Maimonides», The Jewish Quarterly Review 7:2 (Jan. 1895) págs. 346-348.

47 Solomon BAmberger, Maimonides, Moses, 1135-1204. Maimonides' Commentar zum Tractat Challah, zum ersten Male im arabischen Urtext herausgegeben, mit verbesserter hebräischer Uebersetzung, deutscher Uebersetzung, Einleitung und Anmerkungen (Frankfurt am Main: Kauffmann, 1895).

48 Max Bermann, Maimonides, Moses, 1135-1204. Maimonides' Commentar zum Tractat Edujoth. Abschn. I, 1-12, zum ersten Male im arabischen Urtext hrsg., mit verb. hebräischer Uebersetzung, deutscher Uebersetzung, Einleitung, und Anmerkungen vrsehen (Berlin: Itskovski, 1897).

49 Solomon Bamberger, Maimonides, Moses, 1135-1204. Maimonides' Commentar zum Tractat Kilajim, zum ersten Male im arabischen Urtext herausgegeben, mit verbesserter hebräischer Uebersetzung und mit Anmerkungen versehen (Frankfurt am Main: Kauffmann, 1891).

50 Natanel Almoli y Jakob Fromer, Maimonides, Moses, 1135-1204. Maimonides' Commentar zum Tractat Middoth, mit der hebräischen Uebersetzung des Natanel Almoli: kritische Ausgabe mit Anmerkungen und Zeichnungen (Breslau: Th. Schatzky, 1898). 
manera parcial ${ }^{51}$. Al inglés poseemos las de A. David a Abot (1968) ${ }^{52}$; Zvi L. Lampel, que incluye la traducción inglesa del prefacio de alHarizi (1975) ${ }^{53}$; de Rosner (1975) que se ocupa de la introducción a Zeraim y el comentario a Berajot ${ }^{54}$ así como en otra obra unos años más tarde (1981) lo hará con Sanhedrín ${ }^{55}$. P. Forchheimer traducirá el Pirqué Abot dentro de una obra mayor en 1974 que será, más tarde, publicada de nuevo en $1983^{56}$. Al francés la de É. Smilévitch que incluye solamente Abot (1990) ${ }^{57}$.

\subsection{El Kitāb al-farā ’id (1168)}

El Kitāb al-farā'id, conocido por su título hebreo (Séfer ha-Mișvot) o Libro de los Preceptos, fue redactado antes del Mišné Torá y mientras Maimónides escribía su comentario a la Mišná. Pese a que en realidad

51 Leopold Nebenzahl, Maimonides, Moses, 1135-1204. Mischnah-Kommentar zum Tractat Kethuboth (Abschnitt IX-XI). Arabisches Urtext auf Grund von zwei Handschriften zum ersten Male herausgegeben mit verbesserter hebräischer Uebersetzung (Berlin: Itskovski, 1905).

52 Arthur David, Moses Maimonides, The Commentary to Mishnah Aboth. Translated with an Introduction and Notes (New York: Bloch Pub. Co., 1968).

${ }^{53}$ Zvi L. Lampel, Maimonides' Introduction to the Talmud. A translation of the Rambam's Introduction to his Commentary on the Mishnah (New York: Judaica Press, 1975).

54 Fred Rosner, Mosheh ben Maimon. Commentary on the Misnah. Introduction to Sder Zraim and Commentary on Tractate Berachoth (New York: Judaica Press, 1975). Véase también la traducción hebrea de Julius Löwenstein, Maimonides, Moses, 11351204. Maimonides' Commentar zum Tractat Bekhoroth (Berlin: Itskovski, 1897).

55 Fred Rosner, Maimonides' Commentary on the Mishnah. Tractate Sanhedrin. Translated into English with Introduction and Notes (New York: Sepher-Hermon Press, 1981).

56 Paul ForchHeimer, Maimonides' Commentary on Pirkey Avoth (Jerusalem-New York: Feldheim Publishers, 1974), y Maimonides' Commentary on Pirkey Avoth: Living Judaism. The Mishna of Avoth with the commentary and selected other chapters of Maimonides translated into English and supplemented with annotation and a systematic outline for a modern Jewish philosophy (Jerusalem-New York: Feldheim Publishers, 1983).

57 Éric Smilévitch, Moïse Maïmonide, Rachi, Rabbénou Yona. Commentaires $d u$ Traité des Pères. Pirqé Avot (Lagrasse: Éditions Verdier, 1990). 
este sería el primer volumen de esta obra mayor, lo cierto es que ha obtenido una entidad propia mereciendo su propio espacio. Todo apunta a que este tipo de trabajos, basados en enumerar los 613 preceptos del judaísmo sintetizando sus rasgos principales, eran un género común entre los gaones que precedieron a Maimónides. A esta, le antecedieron las propias de Šimeón Qayyara, Saadia Gaón y Šemuel ben Hofni Gaón, Ibn Gabirol, entre otros. Se ha de poner de relieve el papel único que tiene esta obra en la organización del Mišné Torá puesto que esta basa su disposición en los preceptos, de hecho, estos sirven como subtítulos a las diferentes secciones del Mišné Torá, asegurándose así de cubrir toda la cuestión halájica ${ }^{58}$.

Parece ser que esta no fue la única obra que Maimónides dedicó a los preceptos, sino que igualmente se hará eco de esta temática con Hiljot ha-Yerušalmí (Leyes del Talmud palestinense), inacabado, quizá siguiendo los pasos de Alfasi, cuyo comentario del Talmud de Palestina era ampliamente usado en Oriente, sin embargo, abandonó la tarea y el manuscrito se encontró en la Guenizá de El Cairo, como apunta Zvi Stampfer ${ }^{59}$.

58 Moshe Halbertal, Maimonides: Life and Thought (New Jersey: Princeton University Press, 2014) págs. 107-108. Dos buenos estudios en torno a esta obra son los de David Henshre, "'Like a Spring That Becomes Stronger': Maimonides' Sefer haMitzvot and Its Role in His Legal Thought», en By the Well: Studies in Jewish Philosophy and in Halakhic Thought Presented to Gerald Blidstein, eds. Uri EHRLICH et al. (Beer Sheva: Ben Gurion University Press, 2008 [en hebreo]) págs. 151-182 y «On Maimonides' Halakhic Thought: Inner Dynamism versus Institutional Conservatism - On the Nature of Halakha in Maimonides' Sefer ha-Mitzvot» en Maimonides' Conservatism, Originality and Revolution, ed. Aviezer Ravitzky (Jerusalem: Shazar, 2008 [en hebreo]) vol. I, págs. 119-153.

59 Este fragmento, identificado como NS 284.120 en la Taylor-Schechter, fue presentado como fragmento del mes en el año 2011 junto con una introducción de Zvi Stampfer que se había encargado de estudiarla. El enlace a este fragmento puede verse en http://www.lib.cam.ac.uk/Taylor-Schechter/fotm/september-2011/index.html (acceso 25/02/2019). También pueden consultarse las siguientes investigaciones: Saul LiEBERman, The Laws of the Palestinian Talmud of Rabbi Moses ben Maimon (New York: The Jewish Theological Seminar, 1947) [en hebreo]; Simon HopkINs, «A new autograph of Maimonides' Hilkhot Ha-Yerushalmi», Journal of Semitic Studies 28 (1983) págs. 273296; Zvi Stampfer, «Maimonides' Hilkhot ha-Yerushalmi Ketubot», en Jubilee Volume in Honor of Professor Rabbi N. E. Rabinovitz, eds. Zvi Heber and Carmiel CoHEN (Ma'ale Adumim: Ma'aliyot, 2011) págs. 12-108. 
Los traductores que se ocuparon de traducir la obra al hebreo fueron Abraham ben Hasdai en torno a 1230, Solomon ben Yosef ibn Ayyub hacia 1240 y Mošé ibn Tibbón alrededor de $1260^{60}$, siendo esta última la más utilizada en diferentes ediciones como la de A. M. Geller en $1987^{61}$. Otra es la de Qafih en $1971^{62}$. Traducciones a otras lenguas tenemos al castellano en dos volúmenes por Natán Grunblatt en $1985^{63}$; al francés por Bloch en $1888^{64}$; al alemán por M. Peritz (1881) del original árabe junto la traducción también al hebreo y conteniendo únicamente los primeros preceptos ${ }^{65}$; y al inglés por C. B. Chavel en 196766.

\subsection{El Dalālat al-Hāirīn (1190)}

La gran obra filosófica y teológica de Maimónides será, sin duda, su Dalālat al-Hāirīn o Guía de Perplejos, escrita en judeo-árabe en torno al 1190 y revisada pocos años antes de su muerte en 1204. Ya en su introducción, el sabio cordobés se esfuerza en clarificar los objetivos de la composición de su Guía, esto es, dar a conocer la Ley en su verdadero sentido dirigiéndose a aquellos pudieran percibir la contradicción entre el sentido literal de la Ley y la razón ${ }^{67}$.

60 Silver, Maimonidean Criticism, pág. 30.

61 Anne-Marie Geller, Moïse Maïmonide. Le livre des commandements (Lausanne: L'Age d'Homme, 1987).

62 Yosef QAFIH, Mosheh ben Maimon. Book of Commandments (Jerusalem: Mossad Harav Kook, 1971).

63 Natán Grunblatt, Libro de los preceptos, Tomo I: Preceptos positivos. Tomo II: Preceptos negativos (Buenos Aires: Jabad Lubavitch, 1985).

${ }^{64}$ Moïse Bloch, Le livre des préceptes par Moïse ben Maimon dit Maïmonide (Paris: E. Bouillon et E. Vieweg, 1888).

${ }^{65}$ Moritz Peritz, Das Buch der Gesetze von Moseh ben Maimun. Im arabischen Urtexte nebst der hebraischen Uebersetzung des Shelomoh ben Joseph Ibn Ajub zum resten Male herausgegeben und mit Uebersetzung und Anmerkungen versehen (Breslau: Druck von Grass, Barth \& C., 1881) [original árabe, traducción hebrea y alemana que comprende únicamente las primeras reglas].

${ }^{66}$ Charles B. Chavel, The Commandments. Sefer ha-Mitzvoth of Maimonides (London: Soncino, 1967).

67 Sobre la estructura de la Guía, son de destacar los siguientes trabajos de Josef STERn, The Matter and Form of Maimonides' "Guide" (Cambridge: Harvard University 
En cuanto a las traducciones, la principal fue la de Šemuel ibn Tibbón al hebreo ${ }^{68}$ seguida de la de Yehudá al-Harizi ${ }^{69}$ en el siglo XIII, siendo la primera de ellas la que se llevó a cabo en vida de Maimónides. Sin embargo, la labor de Tibbón no parece haber sido únicamente la de verter una obra escrita originalmente a otra, sino que jugó un papel fundamental como mediador entre culturas allanando el camino para la recepción de escritos de Maimónides en las diferentes comunidades de la Europa cristiana, con un bagaje cultural muy distinto de aquel judeoárabe original en el que habían sido compuestas. En este sentido, y como apunta Carlos Fraenkel, Ibn Tibbón mismo fue el primero de una larga lista de comentaristas ya que él mismo añadió numerosas glosas al texto, alrededor de una centena, no únicamente tratando de ilustrar ciertos aspectos del encuentro de Ibn Tibbón con la Guía, sino también sobre el complejo proceso de transmisión del corpus de Maimónides de un contexto cultural a otro ${ }^{70}$.

Press, 2013); Juan Pedro Monferrer SAla, «Modernidad hermenéutica en Maimónides a partir de una exégesis contenida en Dalālat al-Hāàirīn (Morê nebukîim) I, 2», Revista española de filosofía medieval 12 (2005) págs. 73-78; Shlomo PINES, «The Philosophical Purport of Maimonides' Halachic Works and the Purport of The Guide of the Perplexed», Maimonides and Philosophy, International Archives of the History of Ideas 114 (1986) págs. 1-14; Menachem Kellner, «Maimonides' 'Thirteen Principles' and the Structure of the Guide of the Perplexed» Journal of the History of Philosophy 20:1 (1982) págs. 76-84.

68 Véase Yehuda Even-Shmuel, (ed.), Maimonides. Moré ha-nebujim (Jerusalem: Mossad Harav Kook, 1987). Esta no es una edición crítica de la traducción pero sí una vocalizada con sus propios comentarios y cuya labor se alargó durante varios años publicándose en volúmenes separados. La parte I (1-49) se publicó en 1935 (reimpr. 1959), parte I (50-76) en 1938 (reimpr. 1959), parte II (1-24) en 1959, parte II (25-48) y III (1-13) en 1987. Para más información puede consultarse el trabajo de James T. RoBINSON, «Maimonides, Samuel Ibn Tibbon, and the Construction of a Jewish Tradition of Philosophy», en Maimonides After 800 Years: Essays on Maimonides and His Influence, ed. Jay Michael Harris (Cambridge: Harvard University Press, 2007) págs. 291-306.

69 Véase la edición de Leon (Aryeh Leib) Schlossberg y Simon B. Scheyer, Yehuda al-Harizi, Moré ha-nebujim (London-Wien 1851-1879; reimp. Tel-Aviv 1953, 1964, 1984) y la aparecida en Vilna (1929).

70 Carlos Fraenkel, «From Maimonides To Samuel Ibn Tibbon: Interpreting Judaism As A Philosophical Religion», en Traditions of Maimonideanism, ed. Carlos Fraenkel (Leiden-Boston: Brill, 2009) págs. 177-212: 177, 178 y 179; del mismo autor, véase From Maimonides to Samuel Ibn Tibbon: The Transformation of the Dalalat alHairin into the Moreh ha-Nevukhim (Jerusalem: The Hebrew University of Jerusalem, 
La primera edición de esta obra fue la realizada por Salomon Munk, en 3 volúmenes en los que se presentaba el original judeoárabe acompañado de una traducción francesa, notas críticas, literarias y explicaciones y publicada en París a mediados del siglo XIX ${ }^{71}$ y que, más tarde, fue reimpresa en 1964 y reeditada por Verdier en 1979. En 1931 I. Joel se basa en la obra de Munk para su edición ${ }^{72}$. Otras serán las de Y. Qafih y la de H. Atay en la década de los setenta ${ }^{73}$.

Sobre las traducciones, también anteriores a la contemporaneidad, encontramos la de Pedro de Toledo del siglo Xv al castellano, editada por M. Lazar (1989) ${ }^{74}$ y A. J. Escudero (1990) ${ }^{75}$. La siguieron la de Johannes Buxtorf en $1629^{76}$ que lo hace desde la la traducción de Ibn

2007); Y. Tzvi Langermann, «A New Source for Samuel ibn Tibbon's Translation of the Guide of the Perplexed and his Glosses on it» [en hebreo], Pe'amim 72 (1997) págs. 51-74.

${ }^{71}$ Salomon Munk, (ed.), Moïse ben Maimon. Le Guide des Égares. Traité de Théologie et de Philosophy par Moïse ben Raimon, publié par la première fois dans l'original arabe et accompagné d'un traduction français et de notes critiques, littéraires et explications (Paris: A. Franck, 1850-1866, reimp. Osnabrück: Zeller, 1964. 3 vols.).

72 Isachar Joel, (ed.), Maimonides. Dalālat al-Hāirīn (Jerusalem: Magnes Press, 1931), con el texto de Munk en caracteres árabes.

73 Yosef QAFIH, Rabbenu Mošé ben Maimón. Moré ha-nebujim (Jerusalem: Mossad Harav Kook, 1972 [en hebreo]), edición y traducción al hebreo, y Huseyn ATAY, Delâletü'l-Hâirîn: Filosof Mûsa ibn Meymûn el Kurtubî (Ankara: Ankara University Divinity Faculty, 1974).

${ }^{74}$ Moshe Lazar, (ed.), Maimonides, Guide for the Perplexed. A 15th Century Spanish Translation by Pedro de Toledo (Mss. 10289, BN Madrid) (Culver City: Labyrinthos, 1989).

75 Antonio J. Escudero Ríos, (ed.), Mose ben Maymon. Guía de Perplejos: Mostrador e Enseñador de los Turbados (Madrid: Kaher, 1990), edición facsímil del Mss. 10289 de la Biblioteca Nacional de Madrid.

76 Proponitur, Maimonidis More Nevochim typis mandandum lingua Arabica, qua $a b$ authore primáo scriptum est. Suscipiendi operis causce sunt, I. Latina editione Buxtorfiana ante multos annos prorsus distracta \& absumpta, iste liber (ad explicandas $S$. Scripturas Apprime utilis) hodie quovis pretio non est redimendus. II. Quamvis clarissimi Buxtorfii versio Latina (si modo haberi posset) maxima ex parte sit accurata, alicubi tamen est justo laxior, \& alibi aliquando virum doctissimum fefillit linguce Hebraica amphibolia, cum non esset originalem textum Arabicum consulendo. Cum itaque doctrinam dicto libro contentam... Si autem tales notce censeantur sacere ut hoc opus in nimiam molem excrescat, his omnibus omissis, nudus textus Arabice \& Latine emittatur. De his autem penes hujus operis promotores judicium esto. Dicti operis sequitur hujus- 
Tibbón al latín. También al latín es la de Agustín Justiniano que es incluso anterior a la de Buxtorf, en 1520, basándose en la versión de alHarizi y que fue reimpresa en el siglo XVII ${ }^{77}$. Al inglés, destacamos las de M. Friedländer en 3 volúmenes a finales del XIX ${ }^{78}$; aquella de J. Guttmann y Rabin en $1952^{79}$; la de Shlomo Pines en $1963^{80}$. Al alemán y también basado en la de Ibn Tibbón, lo hacen R. Fürstenthal ${ }^{81}$, M. Stern $^{82}$ y S. Scheyer ${ }^{83}$ a mediados del siglo xIX y Weiss en el $\mathrm{XX}^{84}$. Al francés y entre 1850-1866 encontramos la traducción francesa de David Kaufmann en su obra Geschichte der Attributenlehre ${ }^{85}$ desde el original árabe estudiado por Salomon Munk. Al italiano conocemos la de David J. Maroni en 1870 y la de Mauro Zonta en época más reciente (2004) ${ }^{86}$. Al húngaro y acompañada de notas, la tradujo Klein unos años después, en 1878. Al hebreo moderno podemos encontrar la de Qafih que indica-

modi Specimen (Oxford 1690); Johannes Buxtorf, Rabbi Mosis Maimonidis liber Doctor Perplexorum (Basilea: Jo. Jacob Genath, 1629, siguiendo la traducción de Ibn Tibbón; reimp. 1969).

77 Rabbi Mossei Aegyptii Dux seu director dubitantium aut Perplexorum in tres libros divisus et summa accuratione Rev. P. Augustini Iustiniani ordiris Praedicatorii Nebiensium Episcopum recognitus (Paris: Badius Ascensius, 1520, basándose en la traducción de al-Harizi; reimp. 1964).

78 Michael Friedländer, The Guide for the Perplexed by Moses Maimonides (London: Trübner and Co., 1881-1885), en 3 vols.

79 Julius Guttmann y Haim Rabin, Moses ben Maimon. The Guide of the Perplexed. An abridged Edition with Introduction and Commentary (London: East and West Library, 1952).

80 Shlomo Pines, The Guide of the Perplexed (Chicago: University of Chicago Press, 1963-1969), en 2 vols.

81 Rafael Fürstenthal, Moré Nebujim (Krotoschin 1893), conteniendo solo la primera parte.

82 Emanuel Stern, Moré Nebujim (Vien: Verlag von J. Schlossberg's Buchhandlung, 1864), conteniendo solo la segunda parte.

83 Simon SCHEYER, Zurechtweisung der Verirrten (Frankfurt am Main: Ferdinand Hauch, 1838), conteniendo solo la tercera parte.

${ }^{84}$ Adolf WeIss, Mose ben Maimon. Führer der Unschlüssigen (Leipzig: Meiner, 1923; reimp. Hamburg 1972 y 1995, con introducción de Johann MAIER).

85 David Kaufmann, Geschichte der Attributenlehre (Gotha: Perthes, 1877).

${ }^{86}$ Mauro ZonTA, Mosè Maimonide. La Guide dei perplessi (Torino: Editrice Torinesa, 2004); David Jacob Maroni, Guida degli Smarrini (Firenze: I. Costa, 1870). 
mos con anterioridad, la de Yosef ben Shlomo en $1977^{87}$, la de Michael D. Schwarz en $1996^{88}$ que, unos años después, publicó una nueva traducción hebrea ${ }^{89}$. Al castellano, observamos las de J. Suárez (1935) ${ }^{90}$, Fernando Valera $(1946,1988)^{91}$, B. Garzon $(1947)^{92}$, L. Duvojne (1955) ${ }^{93}$ y D. Gonzalo Maeso (1984 y reimpresa en 1994) ${ }^{94}$. Pese a que no sea el objetivo de las páginas, una comparación entre estas traducciones al castellano podría resultar muy interesante. Al catalán tenemos la de Eduard Feliu, en 1986, no solo de la Guía sino de otros escritos ${ }^{95}$.

Conocemos la intención del orientalista Edward Pococke de hacer una edición conjunta de la Guía en judeoárabe, hebreo y latín en Constantinopla, pero finalmente decidió no llevarla a cabo ${ }^{96}$. Por último, la

87 Yosef Ben Shlomo, Rabbenu Mosheh ben Maimon. Moré Nebujim (Jerusalem: Mossad Bialik, 1977).

88 Michael D. Schwarz, Maimonides' Guide of the Perplexed, translated from Arabic into Hebrew and provided by Notes and Indices (Tel-Aviv: Tel Aviv University Press, 1996).

89 Michael D. Schwarz, Moré Nebujim (Tel Aviv: Tel Aviv University Press, 2002) en 2 vols., en la que Schwartz provee de una nueva traducción hebrea así como referencia la literatura secundaria pasaje por pasaje.

90 José SuÁrez Lorenzo, Guía de Descarriados, Tomo I (Madrid: Instituto Maimónides, 1935).

91 Fernando VAlera, (trad.), Maimónides. Guía de los descarriados (México: Orion, 1946) contenía únicamente capítulos seleccionados, teniendo que esperar hasta la década de los ochenta para una traducción completa (Madrid: Editorial Barath, 1988). En los últimos años se ha vuelto a editar como Guía de Perplejos o descarriados (Barcelona: Ediciones Obelisco, reimpr. 2010).

92 Baruj Garzon, Guía de Descarriados. Tratado del Conocimiento de Dios, traducción castellana, selección y estudio de Fernando Valera (México: Orion, 1947; reedición con prólogo, Madrid: Barath, 1988).

93 León Dujovne, Guía de Descarriados (Buenos Aires: Editorial Sigal, 1955. 3 vols.), que es la primera con el texto íntegro después de la de Pedro de Toledo.

94 David Gonzalo Maeso, (ed.), Guía de perplejos (Môrèh nebûkîm) Rabbi Mosé Ben Maimón (Maimónides) (Madrid: Editora Nacional, 1984; reed. Madrid: Trotta, 1994).

95 Maimónides. De la Guia dels perplexos i altres escrits. Traducció i edició (Barcelona: Editorial Laia, 1986).

96 Gerald James ToOmer, Eastern Wisedome and Learning: The Study of Arabic in Seventeenth-century (New York: Clarendon Press of Oxford University Press, 1996) pág. 135. Sobre Edward Pococke, véase el último estudio de Claire GallieN, «Orienta- 
más reciente obra en torno a la historia de las traducciones de la Guía, desde el siglo XIII hasta el XX, es la editada por J. Stern, J. T. Robinson y Y. Shemesh a través de las contribuciones de destacados especialis$\operatorname{tas} 97$.

\subsection{El Mišné Torá (1178)}

El Mišné Torá, Yad Hazaqá o Segunda Ley es, dentro de la categorización de las obras de jurisprudenca rabínica, el trabajo de Maimónides por excelencia. Escrito en hebreo ${ }^{98}$, hecho que ya lo diferencia del resto de corpus, es una sistematización de la halajá judía a la que acompañan el comentario y la interpretación a lo largo de los catorce libros de los que se compone. De todos ellos, los dos primeros, el Libro del Conocimiento y el Libro del amor, son los que mayor interpretación filosófica contienen y han sido estudiados en numerosas ocasiones de forma aislada. Ejemplo de ello son las ediciones y traducciones de S. Liebermann

list Pococke, Brokering across Borders, Disciplines and Genres», en The Internationalization of Intellectual Exchange in a Globalizing Europe 1636-1780, ed. Robert MANKIN (London: Bucknell University Press, 2018) págs. 1-29.

97 Maimonides' Guide of the Perplexed in Translation. A History from the Thirteenth Century to the Twentieth (Chicago-London: University of Chicago Press, 2019).

98 Véase el reciente trabajo de Ariel CHAnAN, «Usage of Biblical Vocabulary in Mishneh Torah (The Code of Maimonides)», Iberia Judaica 7 (2015) págs. 127-140, o los de Yehuda Halper, «Does Maimonides's Mishneh Torah Forbid Reading the Guide of the Perplexed? On Platonic Punishments for Freethinkers», AJS Review 42:2 (2018) págs. 351-379 y David GiLlis, Reading Maimonides' Mishneh Torah (Oxford: Littman Library of Jewish Civilization, 2015). Esta última obra es interesante porque propone que la estructura del Mišné Torá trataría de emular aquella del universo cósmico en diez esferas y cuatro elementos que se corresponderían con los catorce libros de los que consta. La revisión en torno a esta consideración, de hecho, la realiza Yehuda HaLPER («David Gillis, Reading Maimonides’ Mishneh Torah. Oxford: Littman Library of Jewish Civilization, 2015. 448 pp», AJS Review 41:1 [2015] págs. 252-254). Otros trabajos interesantes sobre esta obra son los de David Henshke, «Agency and Divorce Proceedings: On the Legal Methodology of Maimonides' Mishne Torah», Jewish Law Annual 14 (2003) págs. 163-191; Menachem KellneR, «The Literary Character of the Mishneh Torah: On the Art of Writing in Maimonides' Halakhic Works», Me'ah She'arim: Studies in Medieval Jewish Spiritual Life in Memory of Isadore Twersky, eds. Ezra FleISCHER et al. (Jerusalem: Magnes Press, 2001) págs. 29-45. 
en $1964^{99}$, Y. Qafih en 1964 en la que incluye manuscritos yemeníes ${ }^{100}$, M. Hyamson (1974) ${ }^{101}$, de V. Nikiprowetzky y A. Zaoui (1985) ${ }^{102}$, S. Z. Havlin de $1997^{103}$, y A. Steinsaltz en años más recientes (2014) ${ }^{104}$.

Las ediciones del Mišné Torá son, dada la importancia que reviste la obra en sí misma, numerosísimas. La más destacada por la gran cantidad de variantes que recoge y la labor de recopilación de los comentarios más importantes anotados en su bibliografía es la de S. Frankel (2000) ${ }^{105}$, aunque hay otras como las de P. Birnbaum (1944) 106, M. Rabinowitz $(1985)^{107}$, Z. H. Freizler (1986) ${ }^{108}$, N. Rabinowitz (2011) ${ }^{109}$, Y. Makbili (2013) ${ }^{110}$.

99 Saul Liebermann, (ed.), Mishneh Torah: The Book of Knowledge (Jerusalem: Mossad Harav Kook, 1964).

100 Yosef QAfin, (ed.), Mishneh Torah: The Book of Knowledge (Jerusalem: Mossad Harav Kook, 1964).

101 Moses Hyamson, (trad.), Maimonides: The Book of Knowledge (Jerusalem: Feldheim, 1974).

102 Valentin Nikiprowetzky y André ZAoui, Moïse Maïmonide. Le livre de la connaisance (Paris: Balzac, 1985).

103 Shlomo Zalman Havlin, (ed.), Mishneh Torah: Book of Knowledge and Book of Love (Jerusalem-Cleveland: Ofeq Institute, 1997).

104 Adin Steinsaltz, Mishneh Torah: The Book of Knowledge and Book of Love (Jerusalem: Shefa, 2014).

105 Shabse Frankel, (ed.), Mišné Torá (Jerusalem: Shabse Frankel, 2000). En el año 2017 se publicó su 16. ${ }^{\mathrm{a}}$ edición.

106 Philip Birnbaum, Maimonides'Mishneh Torah (Yad Ha-Hazakah). Edited from rare manuscripts and early texts, vocalized, annotated and provided with an introduction (New York: Hebrew Pubishing Co., 1944). 1985).

107 Mordecai Rabinowitz, (ed.), Mišné Torá (Jerusalem: Mossad Harav Kook,

108 Z. H. Freizler, (ed.), Séfer Mišné Torá (Jerusalem 1986).

109 Nahum Rabinowitz, Mishneh Torah by Rabbi Mosheh ben Maimon (Maimonides). According to Oxford Ms. 613 with a comprehensive commentary (Jerusalem: Ma'aliyot Press, 2011).

110 Yohai Makbili, (ed.), The Code of Maimonides: The Complete Restatement of the Oral Law (Haifa: Or Vishua, 2013). Anteriormente ya había sido publicada en 2009 (Haifa: Or Vishua). 
Sobre las traducciones antiguas, conocemos el Porta Mosis de E. Pococke al latín (1655) ${ }^{111}$ y al portugués por S. da Sylva (1613) ${ }^{112}$. Al inglés, en los años 60 y 70, la editorial de la Universidad de Yale (Yale University Press) en la serie Yale Judaica Series publicó una serie de volúmenes dedicados a la traducción de varios libros del Mišné Torá al inglés cuya labor recayó en diferentes investigadores. Así, de ellos se ocuparon S. Gandz y H. Klein (1961) sobre el Libro de las Estaciones ${ }^{113}$; del Libro de Jueces lo hizo A. M. Hershman (1977) ${ }^{114}$ que en 1949 ya había publicado una traducción del mismo libro y del de mujeres y la agricultura I. Klein (1972 y 1979) ${ }^{115}$. Otras son las de L. Nemoy (1972) ${ }^{116}$ y la de de I. Twersky (1976) ${ }^{117}$. Al español se tiene constancia de las llevadas a cabo por D. Cohen de Lara (1991). Al alemán por J. Maier (2000) ${ }^{118}$ que, anteriormente, ya había seleccionado una muestra de la obra publicándola en otro volumen ${ }^{119}$.

111 Bab Musi, Porta Mosis, sive, Dissertationes aliquot áa R. Mose Maimonide: suis in varias Mishnaioth, sive textus Talmudici partes, commentariis promissce, quae ad universam seráe Judceorum disciplinam aditum aperiunt: nunc primáum Arabicáe prout ab ipso autore conscriptce sunt, \& Latine edita: unáa cum Appendice notarum miscellanea / operãa \& studio Edvardi Pocockii. Ed. Oxonice Excudebat H. Hall. Impensis R. Davis, 1655. Maimonides, Mishneh Torah. Latín: Canones poenitentiae Hebraicáe á R. Mose. Egyptio descripti, latinitate donati á G.N. (Cantabrigiae: T. and I. Buck, Academiae celeberrimae typographeo, 1681).

112 Samuel da Sylva, Livro intitulado thesuba que he contrition, en el qual se tratan todos modos Della compuesto por o admirable Rabi Moseh do Aegypto (Francafort 1613).

113 Solomon Gandz y Hyman KLeIn, The Code of Maimonides: The Book of Seasons (New Haven: Yale University Press, 1961).

114 Abraham M. Hershman, The Code of Maimonides: The Book of Judges (New Haven-London: Yale University Press, 1977).

115 Isaac KLeIN, The Code of Maimonides: The Book of Women (New Haven-London: Yale University Press, 1972); The Code of Maimonides: The Book of Agriculture (New Haven-London: Yale University Press, 1979).

116 Leon Nemoy, The Code of Maimonides: The Book of Women (New Haven: Yale University Press, 1972).

117 Isadore Twersky, The Mishneh Torah of Maimonides (Jerusalem: The Israel Academy of Sciences and Humanities, 1976).

118 Johann MAIER, Kriegsrecht und Friedensordnung in juedischer Tradition (Stuttgart: Verlag W. Kohlhammer, 2000).

119 Johann MAIER, Friedensordnung und Kriegsrecht im mitteralterlichen Judentum. Dargestellt auf der Basis der Schriften des Maimonides (Barsbüttel: Institut für Theologie und Frieden, 1993). 


\subsection{Obras atribuidas}

Pese a la extensa producción de Maimónides, existen algunas obras atribuidas a él sobre las que, al parecer, no hay consenso. Si bien la hipótesis de su no autoría en algunas de ellas se muestra más probada que en otras, lo cierto es que la mayoría de los investigadores prefieren no cerrar la cuestión por completo a la espera de nuevas evidencias que puedan corroborar o desechar sus apreciaciones. Este es el caso, por ejemplo, de Y. Tzvi Langermann que, en lo que respecta al Maamar ha-yihud (Tratado sobre la Unidad), argüía que Maimónides podía no ser el autor pero el interrogante debía dejarse abierto ${ }^{120}$.

Cuatro son las obras atribuidas, no confirmadas, a Maimónides: $M a-$ amar ha-yih̆ud, Tiša Peraquim mi-yihud (Nueve capítulos de la Unidad), Peraquim ba-hașlajá (Capítulos de la felicidad, traducido usualmente por Tratado sobre la Felicidad), y un comentario al libro de Job ${ }^{121}$ y cuatro han sido los investigadores que más se han dedicado a estos: Herbert A. Davidson, Y. Tzvi Langermann que mencionamos anteriormente, Fred Rosner y Jacob Dienstag. Los estudios generales que han originado son los de Davidson en 2001 y $2005^{122}$, Langermann en $2001^{123}$ y Rosner en $1991^{124}$.

120 Y. Tzvi Langermann, «A New look at the 'treatise of Unity' Ascribe to Maimonides» [en hebreo], Tarbiz 65 (1995-1996) págs. 109-128. También Davidson, Moses Maimonides: The Man and his Works, pág. 310.

121 Véase Davidson, Moses Maimonides: The Man and his Works, págs. 309-315, donde desglosa estas obras atribuidas.

122 Herbert A. Davidson, «The Authenticity of Works Attributed to Maimonides», en Me'ah She'arim: Studies in Medieval Jewish Spiritual Life in Memory of Isadore Twersky, eds. G. BLIDSTEIN et. al. (Jerusalem: Magnes Press, 2001) págs. 118-125, y Moses Maimonides: The Man and his Works. Puede verse también García ArÉvalo, Cartas de Maimónides, págs. 101-103.

123 Y. Tzvi Langermann, «On Some Passages Attributed to Maimonides», Me'ah She'arim: Studies in Medieval Jewish Spiritual Life in Memory of Isadore Twersky, eds. G. Blidstein, et al. (Jerusalem: Magness Press, 2001) págs. 223-240.

124 Fred Rosner, Six Treatises Attributed to Maimonides (Northvale: Jason Aronson, 1991).

SEFARAD, vol. 79:2, julio-diciembre 2019, págs. 469-496. ISSN: 0037-0894. https://doi.org/10.3989/sefarad.019-014 
Sobre el Maamar ha-yihud, poseemos la edición de M. Steinschneider de $1847^{125}$ y la traducción inglesa de Rosner ${ }^{126}$. En cuanto a Peraquim behașlajá o Tratado de la Felicidad, tenemos las ediciones de Bacher (18961897) ${ }^{127}$, Baneth y Davidowitz (1939) ${ }^{128}$, Lichtenberg (1859) ${ }^{129}$ y dos artículos bibliográficos de J. Dienstag a los que no hay que dejar de prestar atención $^{130}$. La traducción al inglés la ofrece Langermann en $1986^{131} \mathrm{y}$ Dienstag en $1990^{132}$. La traducción al español puede verse en C. del Valle ${ }^{133}$.

\section{RESPONSA DE MAIMÓNIDES: EDICIONES y TRADUCCIONES}

Algunas de las responsas de Maimónides poseen también un carácter filosófico y de jurisprudencia rabínica. Hablamos de la epístola sobre la apostasía y del Yemen, en 1160-1161 y 1174 respectivamente; la que escribe a Hasday ha-Levi en 1190; el Maamar Tehiyat ha-metim (Tratado

125 Moritz STEInSCHNEIDER, Schene ha-Meoroth enhält Maamar ha-Jichud (Abhandlung ueber die inheit), aus dem arabischen des R. Moses ben Maimon, hebraeisch von R. Isaak b. Natan, zum resten Mal herausgegeben (Berlin: L. Zarenzanski, 1847).

126 Fred Rosner, The Existence and Unity of God. Three Treatises Attributed to Moses Maimonides. Translated and annotated from the Hebrew Editions (Northvale: Jason Aronson, 1990).

127 Wilhelm BACHER, «The Treatise on Eternal Bliss attributed to Moses Maiminu», Jewish Quarterly Review 9 (1896/7) págs. 270-289.

128 David H. Baneth y Harry S. Davidowitz, De beatitude (Jerusalem: Nezike Nirdamim, 1939) [en hebreo].

129 Lichtenberg, Collection of the Response.

130 Véase Jacob DienstaG, «Perakim be-Hazlahah Attributed to Maimonides: A Bibliography of Editions, Translations, and Studies», Studies in Bibliography and Booklore 16 (1986) págs. 51-56; del mismo autor, «The Treatise on Eternal Bliss - Bibliography», en Six Treatises Attributed to Maimonides, ed. Fred Rosner (Northvale: Jason Aronson, 1991) págs. 57-64.

131 Y. Tzvi Langermann, «A Renewed Examination of Ma'amar ha-Yihud, Attributed to Maimonides» [en hebreo], Tarbiz 65 (1986) págs. 109-128.

132 Jacob Dienstag, «Ma'mar Hayichud (Treatise on the Unity of God) - Bibliography», en The Existence and Unity of God: Three Treatises Attributed to Moses Maimonides, ed. Fred Rosner (Northvale: Jason Aronson, 1990) págs. 89-94.

133 Carlos Del Valle, Cartas y testamento de Maimónides (1138-1204) (Córdoba: Caja de Ahorros de Córdoba, 1989) págs. 253-271. 
de la Resurrección de los muertos) en 1191, que fue traducida al hebreo por Šemuel ibn Tibbón en tanto que la original judeo-árabe era la usada por las comunidades judías yemeníes; las que envía a Rabí Yosef bar Yehudá ben Simeón entre 1190 y 1191; la dirigida al dayán de Alejandría; a Rabí Yonatán ha-Cohen de Lunel y a los sabios de la comunidad de Lunel (1199-1200) ${ }^{134}$ o a los de Montpellier (1194); a sus discípulos Yosef ibn Gabir y Yosef ibn Aknin; su tratado sobre el calendario o la carta a su hijo Abraham, cuyo seguimiento sobre sus ediciones y traducciones junto con una descripción de cada una de ellas fue publicado en $2009^{135}$.

Esta correspondencia ya abrió un abanico de ediciones de mano de $\mathrm{Z}$. H. Edelmann (1856), A. Lichtenberg (1859) y Y. Goldmann (1877) ${ }^{136}$ aunque también poseemos las de D. H. Baneth (1946) en hebreo siendo con posterioridad reeditada en inglés en 1985 y que se configuró como una de las obras de referencia ${ }^{137}$. Entre 1957 y 1986, aparece la importante edición de J. Blau en cuatro volúmenes con anotaciones ${ }^{138}$. A esta le siguió en 1953 la de Lewin ${ }^{139}$ y desde 1957 hasta 1988 asistimos a la aparición de cinco ediciones como son las de M. D. Rabinowitz (1957) ${ }^{140}$, A. Halin $(1970)^{141}$, Y. Qafih (1972) ${ }^{142}$, S. D. Goitein (1973) ${ }^{143}$ e I. Shailat (1995) ${ }^{144}$

${ }^{134}$ Uno de los últimos estudios sobre viene de mano de Charles H. SHEER, Maimonides' Gran Epistle to the Scholars of Lunel: Ideology and Rhetoric (Boston: Academic Studies Press, 2019).

135 García Arévalo, Cartas de Maimónides.

136 Zvi H. Edelman, Hemdah Genuzah (Königsberg: Gruber and Gruphrat, 1856); Lichtenberg, Collection of the Response; Y. Goldmann, Iguerot.

137 David H. Baneth, Epistulae (Jerusalem: Mekize Nirdamim, 1946).

138 Joshua Blau, Maimonides' Responsa, 4 vols (vols. 1-3, Jerusalem: Mekize Nirdamim, 1957-1961; vol. 4, Jerusalem: Rubin Mass, 1986) [en hebreo].

139 B. Lewin, Iguerot.

140 Mordecai D. Rabinowitz, Letters of Maimonides (Jerusalem: Mossad Harav Kook, 1957; 7. a edición, 1974) [en hebreo].

141 A. Halin, Letters and Biography (Tel Aviv: Ed. M. Bar-Joseph, 1970).

142 Yosef QAfIH, Letters (Jerusalem: Mossad Harav Kook, 1972) [en hebreo].

143 Shlomo Dov GoIteIn, Letters on Medieval Jewish Traders (Princeton: Princeton University Press, 1973).

144 Isaac ShaILAT, The Letters and Essays of Moses Maimonides (Jerusalem: Shailat Publishing, 1995) [en hebreo]. 
Las cartas han sido vertidas en su mayoría al inglés por I. Abrahams (1926) ${ }^{145}$, A. Ya'ari (1935) ${ }^{146}$, F. Kobler (1952 y 1954) ${ }^{147}$, R. L. Weiss y C. E. Butterworth (1975) ${ }^{148}$, L. D. Stitskin (1977) ${ }^{149}$, la obra conjunta de A. Halkin y D. Hartman (1985) ${ }^{150}$, J. A. Kraemer (1998) ${ }^{151}$ y N. Roth (1985) ${ }^{152}$. Únicamente disponemos de una traducción en francés de J. de Hulster (1983) ${ }^{153}$. En español poseemos tres obras básicas para el estudio de las cartas de Maimónides como son las de J. Targarona (1987) ${ }^{154}$, la obra conjunta de M. ${ }^{a}$ J. Cano y L. Ferre (1988) ${ }^{155}$ y C. del Valle (1989) ${ }^{156}$.

145 Israel Abrahams, Hebrew Ethical Wills (Philadelphia: The Jewish Publication Society, 1926).

146 Abraham YA‘ARI, «Ha-Rambam be-“iggerotaw», Moznayim 3 (1935) págs. 534 y ss.

147 Franz Kobler, Letters of Jews through the Ages (London: Ararat Publishing Society, 1952) y A Treasury of Jewish Letters (Philadelphia: The Jewish Publication Society, 1954).

148 Charles E. Butterworth, Ethical Writings of Maimonides (New York: New York University Press, 1975).

149 Leon D. STITSKIN, Letters.

150 Abraham Halkin y David Hartman, Crisis and Leadership: Epistles of Maimonides (Philadelphia-New York-Jerusalem: The Jewish Publication Society, 1985).

151 Joel L. Kraemer, «Four Genizah Letters concerning Maimonides», en Mas'at Moshe: Studies in Jewish and Islamic Culture presented to Moshe Gil, eds. Mordecai A. Friedman, Ezra Fleischer y Joel A. Kraemer (Jerusalem: The Bialik Institute, Chaim Rosenberg School of Jewish Studies, Tel-Aviv University, 1998) págs. 381-400.

152 Norman Roth, Maimonides: Essays and Texts: 850th anniversary (Madison: Hispanic Seminary of Medieval Studies, 1985).

153 Jean de Hulster, Epîtres. Epître sur la persecution. Epître au Yemen. Epître sur la résurrection. Introduction au chapitre Heleq (Lagrasse: Éditions Verdier, 1983).

154 Judit Targarona, Sobre el Mesías: Carta a los judios del Yemen; Sobre astrología: Carta a los judios de Montpellier (Barcelona: Riopiedras, 1987).

155 María José Cano y Lola Ferre, Cinco epístolas de Maimónides (Barcelona: Riopiedras, 1988).

156 Cartas y testamento. 


\section{Consideraciones Finales}

El caudal de estudios en torno a Maimónides y su obra es de difícil cuantificación. Sin embargo, también lo es el que tiene como objetivo el análisis de las traducciones coetáneas de sus obras. Nos referimos a aquellas traducciones llevadas a cabo por la familia Ibn Tibbón, sobre todo, Šemuel ibn Tibbón y Mošé ibn Tibbón, al-Ḥarizi y otros traductores de los siglos XIII y XIV. Sería necesario tratar de paliar el vacío existente en torno a estudios que compararan las versiones de estos traductores medievales ya que, individualmente, han sido estudiados copiosamente pero también sería interesante volver a la cuestión de los traductores menores, los menos analizados ya que no encontramos sino apenas alguna edición aislada.

El siglo XVII, por su parte, destaca por las grandes traducciones al latín. Aquellas de Pococke, Buxtorf, entre otros, parecen copar el escenario académico a través de dos obras, el Mišné Torá y el comentario a este, siendo prácticamente nula la actividad destacable en latín en otras obras. Aunque en el siglo XIX sí parece demostrarse cierto interés por las obras que aquí nos ocupan, sobre todo por el 'Tratado de lógica' y los comentarios a tratados independientes del comentario a la Mišná, lo cierto es que la segunda mitad del siglo xx es el gran momento de las ediciones y las traducciones. Hablamos de traducciones a un gran número de lenguas, no solamente al inglés como venía sucediendo, sino también al francés, al italiano, al español, entre otros, ya que las hebreas restringían el círculo de su uso solamente a un público muy limitado que conocía esta lengua, por lo que la necesidad de apertura de este escenario parecería, en principio, cubierta con la numerosa cantidad de traducciones a otras lenguas que aparecen. Esto nos llevaría a otro punto y es el de ver el modo en que los especialistas que no conocen el árabe o el hebreo trabajan sobre Maimónides y sus obras, esto es, qué traducciones son las que más utilizan y, en este caso, qué dificultades encuentran en ellas o los vacíos que les suponen en sus análisis.

Debido al auge que comentamos, muy probablemente, los artículos de Lachterman y Ben-Shammai de 1990 y 1991, respectivamente, respondían a esta inquietud dada la actividad que se vivía en los círculos académicos sobre Maimónides y su legado en este momento. 
En el momento actual, los estudios sobre el legado de Maimónides continúan, pero las ediciones son cada vez más inusuales, algo sobre lo que se debería volver de nuevo. Nos hallamos en el período de estudio, de comparación y análisis con otros autores, otras obras, fuentes e influencias siguiendo con la línea investigadora iniciada en el siglo Xx.

Recibido: 08/03/2019

Aceptado: 04/10/2019 